\title{
Wird ADHS überdiagnostiziert?
}

\author{
Interviewpartner: Brigitte Hackenberg • Christian Kienbacher
}

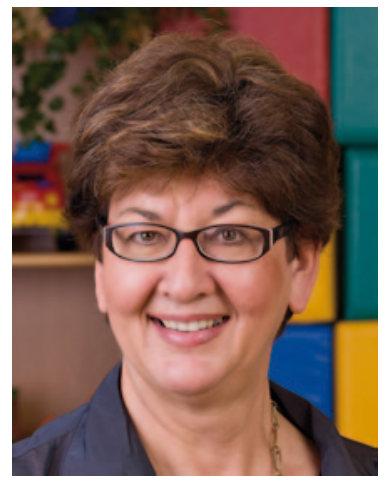

Ass.-Prof. Dr. Brigitte Hackenberg, Universitätsklinik für Kinder- und Jugendheilkunde, Wien.

(c) MedUni Wien

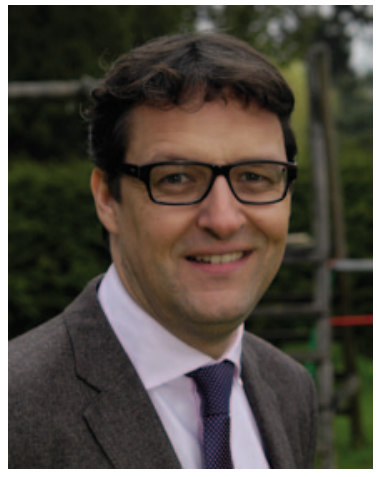

Prim. Dr. Christian Kienbacher, Ambulatorium für Kinder- und Jugendpsychiatrie Floridsdorf, Wien. (C) Privat

Das Aufmerksamkeitsdefizits-Hyperaktivitätssyndrom ADHS ist eine Erkrankung, deren Bekanntheit sich erst in den letzten Jahrzehnten durchgesetzt hat. Gefolgt ist ein regelrechter Boom der für Eltern annehmbaren und für das Umfeld gefälligen Diagnose. Ob eine Überdiagnostik betrieben werde, diskutieren für neuropsychiatrie, vereinigt mit psychiatrie \& psychotherapie Prof. Dr. Brigitte Hackenberg, Universitätsklinik für Kinder- und Jugendpsychiatrie, Wien und Prim. Dr. Christian Kienbacher, Ambulatorium für Kinder- und Jugendpsychiatrie Floridsdorf, Wien.

Neuropsychiatrie: Können Sie die heute wichtigsten Diagnosekriterien von ADHS zusammenfassen und aktuelle Zahlen zur Epidemiologie nennen?

Kienbacher: Die Diagnosekriterien von ADHS sind nicht einheitlich, zwei große Diagnosesysteme bieten unterschiedliche Definitionen an - das verzerrt den Blick auf die Epidemiologie etwas. Die angloamerikanischen DSM-Kriterien haben mit dem Update von DSM IV auf V eine Ausweitung der Alterseinschränkung bis zum 12. Lebensjahr gebracht. Eine Adaptierung, die der Sache meiner Meinung nach nicht zuträglich ist, da der Kontext zur Entwicklung der Kinder abgeschwächt wird. Die in Österreich gebräuchliche ICD-10 Klassifikation beschreibt ADHS als Entwicklungsstörung besser, hier wird eine Erstmanifestation bis zum 6. Lebensjahr gefordert. Zentrale Kriterien bei ICD-10 sind Unaufmerksamkeit, Überaktivität und Impulsivität. Diese müssen sich über mindestens 6 Monate situationsübergreifend, also nicht etwa nur zu Hause, oder nur in der Schule zeigen und von überdurchschnittlicher Ausprägung sein. Betroffene haben eine Filterstörung und können zwischen sensorischen Außenreizen nicht filtern und werten, was in der jeweiligen Situation wichtig ist. ICD-10 differenziert zwischen der einfachen Aktivitäts- und Aufmerksamkeitsstörung F90.0, der hyperkinetischen Störung des Sozialverhaltens F90.1 und der Aufmerksamkeitsstörung ohne Hyperaktivität F98.8. Demgegenüber trennt DSM eine vorwiegend hyperaktive von einer vorwiegend aufmerksamkeitsgestörten Form von ADHS. Die Epidemiologie wird auch durch einen weiteren Aspekt verzerrt: In nur etwa einem Drittel der Fälle tritt ADHS alleine auf, der Rest ist mit eine Reihe von Co-Morbiditäten wie Angststörungen, oppositionellen oder affektiven Störungen assoziiert. Da Störungen des Sozialverhaltens oft gemeinsam in Studien untersucht werden, ergeben sich Prävalenzraten für ADHS zwi- 
schen 2 und $10 \%$ der Gesamtbevölkerung. Realistisch schätze ich in Österreich einen Wert zwischen 2 und $4 \%$ ein. In der Verordnungsrate für Methylphenidat haben wir mit bis $0,6 \%$ in Österreich und Frankreich sehr niedrige Frequenzen, in Deutschland liegen diese bei $3 \%$, in der Schweiz bei $4 \%$. Hier muss aber einbezogen werden, dass gerade bei uns sehr ausbalanciert therapiert wird und viele Kinder von nicht-medikamentösen Behandlungen profitieren.

Hackenberg: International und auch im deutschen Sprachraum setzt sich langsam eine vermehrte Beachtung der Co-Morbiditäten durch, das Interesse gilt nicht mehr ausschließlich den Kernsymptomen. Auch den lange gegenüber Auffälligkeiten im Sozialverhalten unbeachteten emotionalen Störungen und Symptomen wird nun Aufmerksamkeit geschenkt. Die Revision der DSM-Kriterien hat den gegenseitigen Ausschluss zum Autismus relativiert - ich gehe davon aus, dass auch die nächste Fassung der ICD-Klassifikation diesen Aspekt beinhalten wird, der letztlich in die Diagnosehäufigkeit einspielt.

Neuropsychiatrie: Kürzlich veröffentlichte Daten aus den USA zeigen dort einen signifikanten Anstieg an diagnostizierten Fällen auf und haben eine Diskussion über den Stellenwert von ADHS für die Gesundheit der Bevölkerung und das Gesundheitssystem ausgelöst. Bedeuten steigende Inzidenzen automatisch eine Überdiagnostik und letztlich -therapie?

Hackenberg: Diese Ergebnisse sind das Resultat einer vermehrten Awareness für ADHS. Es gibt aber noch einen positiven Aspekt: ein Anstieg der Inzidenz bedeutet nicht obligat einen Anstieg der Verschreibungshäufigkeit von Medikamenten. Weitaus nicht alle Diagnosestellungen folgern eine medikamentöse Behandlung, sie implizieren lediglich einen Bedarf an Betreuungsstellen. Wir wissen, dass nicht-medikamentöse Angebote weltweit nachhinken.

Kienbacher: Eine Übertragung US-amerikanischer Zahlen in den europäischen Raum ist schwierig, zumal die Affinität zu medikamentösen Therapien in den USA weitaus größer ist. Die Indikation zur Pharmakotherapie wird in ganz Europa weitaus reservierter gesehen. Den Anstieg bzw. die Schwankungen der Inzidenzzahlen kann ich mir psychopathologisch nicht erklären, ich denke die tatsächliche Inzidenz bleibt, wie auch bei anderen Entwicklungsstörungen, weitestgehend gleich.
Neuropsychiatrie: Welche Trends nehmen Sie im deutschsprachigen Raum wahr? Welche Initiativen werden von den Fachgesellschaften gesetzt? Suggerieren die internationalen Zahlen am Ende, dass wir langsam genug Awareness haben?

Hackenberg: Es gibt eine Tendenz zur Überdiagnose, gerade bei Störungen des Sozialverhaltens und dort, wo Strukturmängel da sind. Die Schulen, nicht die Ärzte, drängen zu vermehrten Diagnosen und zur Medikation, wo andere Lösungen gebraucht werden. Die Fachgruppen und die Bemühungen in den berufsgruppenspezifischen Bereichen bewegen sich dagegen klar zur Psychotherapie.

Kienbacher: Die Awareness für ADHS ist in der Tat hoch, vor allem im schulischen Bereich. Die Achtsamkeit der Lehrer ist positiv, kann unter Umständen aber problematisch werden, wenn es zu Fehlzuweisungen kommt. Hier ist die Aufgabe der Kindes- und Jugendpsychiater auch einmal klar zu sagen "Nein, das ist nicht ADHS“, selbst wenn die gesellschaftliche Bereitschaft, diese Diagnose anzunehmen, viel höher ist als bei anderen Entwicklungs- oder Verhaltensstörungen. Dennoch sehe ich ADHS als ein Beispiel für eine gelungene öffentliche Bewusstseinsbildung - ähnlich hohes Engagement, nicht nur der Fachgesellschaften, wäre bei anderen Erkrankungen in der Kinder- und Jugendpsychiatrie wünschenswert. Wohl brauchen wir in Österreich noch mehr Bewusstsein, dass ADHS explizit kinderpsychiatrischer Expertise bedarf und nicht in den Aufgabenbereich der Pädiatrie, (Erwachsenen-)Psychiatrie oder Allgemeinmedizin fällt.

Neuropsychiatrie: Wie kann sichergestellt werden, dass keine Patienten mit einer behandlungsbedürftigen Erkrankung übersehen und gleichzeitig in einzelnen Populationen keine unangebracht positiven Diagnosen gestellt werden?

Hackenberg: Indem auf dem Prinzip der multimodalen Diagnostik bestanden wird! Diese Diagnostik ist aufwändig und darf nicht an eine einzige Berufsgruppe delegiert werden. Dabei sind stets alle beeinflussenden Achsen, also nicht nur die umschriebenen Entwicklungsstörungen bzw. Teilleistungsstörungen, sondern auch Achse 5 nach ICD, die (abnormen) psychosozialen Umstände, also familiäre Risikofaktoren wie Streit oder Stress, einzubeziehen.

Kienbacher: Eine flächendeckende und gut aufgestellte kinder- und jugendpsychiatrische Versorgung mit dieser Expertise und die Multiprofessionalität sichert die Versorgungsqualität. Der Ausschluss anderer Entwicklungsstörungen, die wie ADHS imponieren können, ist ein zentrales Kriterium in der Diagnostik. Das gelingt nicht 
in einer halben Stunde - hier fehlen in Österreich Ressourcen, wir sind einfach zu wenig Fachärzte für Kinderund Jugendpsychiatrie. Ein früher Behandlungsbeginn, dort wo er gebraucht wird, beeinflusst den Verlauf ungemein positiv. Das bedeutet, dass ein für die jeweiligen Altersgruppen geeignetes Therapieangebot bereitstehen muss. Natürlich kann in diesem Bereich auch ein soziales Gefälle nicht geleugnet werden: viele nützliche Angebote kommen nur Kindern zugute, deren Eltern das bezahlen können, etwa einen Kletterkurs, der den unmittelbaren Response zwischen Konzentration und Erfolg hautnah vermittelt, nach dem Motto „wenn ich abgelenkt bin, falle ich runter“. Kinder mit ADHS brauchen genau dieses unmittelbare Feedback, Lob oder Kritik sofort! Das sollten auch die betreuenden Lehrer wissen.

\section{Neuropsychiatrie: Wie beurteilen Sie die} Situation bei Mädchen, bei denen ADHS früher häufig nicht erkannt oder falsch interpretiert wurde?

Hackenberg: Mädchen sind wirklich übersehen worden, jetzt hilft uns die bessere Diagnostik. Die Inzidenzunterschiede sind nicht so groß wie gedacht! Bei Mädchen treten Verträumtheit, introvertierte oder depressive Verhaltensmuster in den Vordergrund und sind wesentlich häufiger als angenommen. In der Diagnostik tragen Mädchen einen - übrigens in allen industrialisierten Ländern gleichen - Nachteil: männliche, expansive und sozial störende Verhaltensmuster werden schneller auffällig, bei Mädchen muss aktiv nachgefragt werden.

Kienbacher: ADHS hat vermutlich schon mit dem Geschlecht zu tun. Wenn Mädchen erkranken, imponieren sie eher unaufmerksam als hyperaktiv. Bei den vorwiegend hyperaktiven Kindern dominieren Buben mit 5:1, bei den vorwiegend aufmerksamkeitsgestörten nur 2:1. Es mag eine kleine Dunkelziffer bei Mädchen geben, ich bin mir aber sicher, dass wir nur sehr wenige übersehen. Zu den Gender-Aspekten kommt, dass sich die Pädagogik grundlegend verändert hat. Gehörten Spielzeugpistole und Taschenmesser vor dreißig Jahren quasi zur Grundausstattung jedes Schulbuben, ist das heute praktisch undenkbar.

Neuropsychiatrie: Das Kriterium ,ever diagnosed ADHS“, also die mindestens einmal im Leben erhobene Diagnose, schickt sich an, Patienten sehr lange anzuhaften - wo sehen Sie einen Bedarf für die Re-Evaluation solcher Diagnosen, auch in Hinblick auf den Übertritt ins Erwachsenenalter? Beeinflusst die Industrie die Häufigkeit von Diagnosestellungen und die Aufrechterhaltung mitunter nicht mehr indizierter Therapien?

Kienbacher: ADHS ist noch nicht so lange etabliert, aber es gibt Erwachsene, die betroffen sind oder waren. Auch die Erwachsenenpsychiatrie beginnt diese Menschen wahrzunehmen. Angesichts der noch kleinen Fallzahlen möchte ich vorsichtig urteilen, aber ich habe den Eindruck gewonnen, dass Erwachsene, die als Kinder und Jugendliche effizient behandelt worden sind, erfolgreicher sozial und beruflich integriert sind. Mit einer Teilleistungs- oder Entwicklungsstörung kann man durchaus gut zurechtkommen, nach dem Motto „mit Schwächen leben und erfolgreich sein!“. Die Ausbildungs- und Berufswahl ist sicherlich entscheidend. Es gibt Berufe, die sich mehr, andere weniger anbieten. Erwachsene mit ADHS sind in kommunikativen oder kreativen Aufgaben nicht selten überdurchschnittlich erfolgreich und fallen durch ihr unkonventionelles Auftreten dort positiv auf. Die Kehrseite ist, dass es Menschen geben wird, die lebenslange Probleme durch die Erkrankung haben werden. Wir wissen, dass unter Häftlingen sehr hohe Raten an ever-diagnosed ADHS zu finden sind. Fest steht: ADHS hört nicht mit dem 18. Geburtstag auf.

Hackenberg: Die klinische Praxis zeigt zwei Meilensteine: erstens, das Alter der endenden Pubertät, zwischen 15 und 17 Jahren mildern sich sehr viele Symptome in Stärke und Ausprägung. Die zweite Grenze liegt um das 20. Lebensjahr. Auch im Erwachsenenalter sind noch etwa $50 \%$ der Betroffenen symptomatisch, aber weitaus nicht immer behandlungsbedürftig. Von einer lebenslangen Etikettierung möchte ich also nicht ausgehen. Im Gespräch mit jungen ADHS-Erwachsenen können wir sehr viele Einblicke in die Erkrankung gewinnen. „Kennen Sie diese Symptome aus Ihrer Kindheit und wie wirken sie sich heute aus?" wäre ein möglicher Zugang. Ein wenig beachteter, aber sehr wichtiger Aspekt ist die Elternschaft bei ehemaligen ADHS-Patienten. Erwachsene, die sich konsolidiert haben und in der Berufswelt erfolgreich sind, zeigen mehr Verständnis für die Bedürfnisse und Anliegen ihrer Kinder. Weniger gefestigte lassen dagegen häufig Erziehungsstrukturen vermissen. Eine Einflussnahme der Industrie nehme ich in Österreich nicht wahr. Es wurde erkannt, dass Manipulation in einem sachlich geprägten Umfeld nicht greift.

\section{Das Gespräch führte Dr. Alexander Lindemeier}

